\title{
Space-resolved dynamics of a tracer in a disordered solid
}

\author{
Thomas Franosch ${ }^{\mathrm{a}, \mathrm{b}}$, Markus Spanner ${ }^{\mathrm{a}}$, Teresa Bauer ${ }^{\mathrm{b}}$, Gerd E. Schröder-Turk ${ }^{\mathrm{a}}$, Felix Höfling ${ }^{\mathrm{c}, \mathrm{d}}$ \\ ${ }^{a}$ Institut für Theoretische Physik, Friedrich-Alexander-Universität Erlangen-Nürnberg, Staudtstraße 7, 91058 Erlangen, Germany \\ ${ }^{b}$ Arnold Sommerfeld Center for Theoretical Physics and Center for Nano Science (CeNS), Fakultät für Physik, Ludwig-Maximilians-Universität \\ München, Theresienstraße 37, 80333 München, Germany \\ ${ }^{c}$ Rudolf Peierls Centre for Theoretical Physics, 1 Keble Road, Oxford OX1 3NP, England, United Kingdom \\ ${ }^{d}$ Max-Planck-Institut für Metallforschung, Heisenbergstraße 3, 70569 Stuttgart and Institut für Theoretische und Angewandte Physik, Universität \\ Stuttgart, Pfaffenwaldring 57, 70569 Stuttgart, Germany
}

\begin{abstract}
The dynamics of a tracer particle in a glassy matrix of obstacles displays slow complex transport as the free volume approaches a critical value and the void space falls apart. We investigate the emerging subdiffusive motion of the test particle by extensive molecular dynamics simulations and characterize the spatio-temporal transport in terms of twotime correlation functions, including the time-dependent diffusion coefficient as well as the wavenumber-dependent intermediate scattering function. We rationalize our findings within the framework of critical phenomena and compare our data to a dynamic scaling theory.
\end{abstract}

Keywords: transport, disordered solids, porous materials, Lorentz model PACS: 66.30.H-, 05.10.-a, 61.43-j, 64.60.Ht

\section{Introduction}

The structural dynamics in glass-forming liquids slows down by orders of magnitude upon cooling or compression eventually leading to a quasi-arrested state or a disordered solid. Conventionally the dynamics close to the glass transition slows down uniformly as manifested in the divergence of a single time scale characterizing the slowest process in the system, referred to as the $\alpha$ process. A coherent theoretical picture for a variety of phenomena has emerged by the mode-coupling theory (MCT) of the glass transition [1], developed by Wolfgang Götze and collaborators in the last 25 years. In particular, this approach has explained how a two-step relaxation process with a nontrivial two-time fractal results from the equations of motion close to a bifurcation point. The mechanism encoded in these equations to yield power laws with exponents that are not simple fractions has no precedence in other areas of physics and yet has been identified as generic in nonlinear integrodifferential equations.

The mathematical properties of the MCT solutions are well understood for single component liquids [2] and mixtures [3]; in particular, it has been shown that the solutions allow a representation in terms of a continuous distribution of relaxation rates [4]. The success of the mode-coupling theory is based on the fact that it not only explains how the slow dynamics emerges in principle, but that it also has provided a series of testable predictions which challenge the physical intuition obtained so far. To mention just a few, MCT coherently explains the physics of suspensions of hard-sphere colloids [5, 6, 7], the reentrant phenomenon in 'attractive colloidal glasses' [8, 9], a pronounced minimum in the light-scattering spectra of supercooled liquids [10, 11, 12], or the composition dependence of the structural relaxation in mixtures [13, 14]. For many years only the standard mode coupling equations have been discussed and attempts to go beyond have been focused on extension for non-spherical molecules [15, 16, 17, 18] and more recently to include shear in colloidal suspensions [19, 20].

The overall success of MCT is encouraging to investigate more complex glass-transition scenarios to obtain a deeper insight into the nature and quality of the approximations involved. A particularly interesting candidate are strongly size-disparate mixtures where structural arrest may occur in several steps. There, the majority component of large particles can undergo a glass transition characterized by a frozen disordered structure, whereas the minority species of smaller size meanders through the emerging network of channels. 
Neutron scattering experiments and molecular dynamics simulations indicate a significant separation of time scales for sodium silicate melts also at finite concentration [21], which could be rationalized within standard MCT calculations [22] later. A similar splitting of relaxation times has been found also for size-disparate soft spheres [23] and Yukawa mixtures [24]. The dynamics within a frozen matrix or nanoporous medium has been studied only recently [25, 26] corroborating an intriguing interplay of several dynamic transitions predicted within an extension of the mode-coupling theory for the dynamics within a disordered matrix [27, 28]. The standard MCT of mixtures appears to qualitatively describe many aspects of the splitting of the dynamics [29], yet ultimately predicts that the structural arrest of the majority and minority particles occurs at the same critical point. The observed peculiarities are rationalized as a precursor phenomenon for the two-step freezing not contained in the standard MCT. Krakoviack's extensions to disordered matrices treats the frozen obstacles and the fluid on unequal footing [27, 30] thereby allowing for multiple transitions. Although both approaches appear to give a satisfactory qualitative picture, theoretical issues remain that are poorly understood. First, the freezing in the disordered solid is accompanied by a divergent length scale suggesting that the transition is driven by an entire hierarchy of length scales rather than the Lindemann length for caging. It appears that MCT in its current form is ill-suited to deal with this phenomenon [1]. This manifests itself in 'spurious longtime anomalies' [30] that, if treated correctly, lead to a change in the exponent of the anomalous transport observed in the mean-square displacement [31].

An alternative approach dealing with the dynamics of a single tracer in a disordered matrix is within the framework of critical phenomena. The localization transition of the tracer is due to an underlying percolation transition of the void space as the density of the matrix is altered. As is well known, this geometric problem leads to a self-similar distribution of clusters [32] entailing a series of scaling predictions. It has been suggested that the dynamics of the Lorentz model shares the same universality class with a random resistor network with powerlaw distributed conductances [33, 34, 35]. The critical dynamics of the Lorentz model in the vicinity of the percolation threshold can be described within a scaling ansatz for the van Hove correlation function [36]. Furthermore, corrections to scaling due to irrelevant scaling variables are not negligible and have been included recently [37, 38].

In this paper we investigate the Lorentz model by extensive computer simulations and show that the mean-

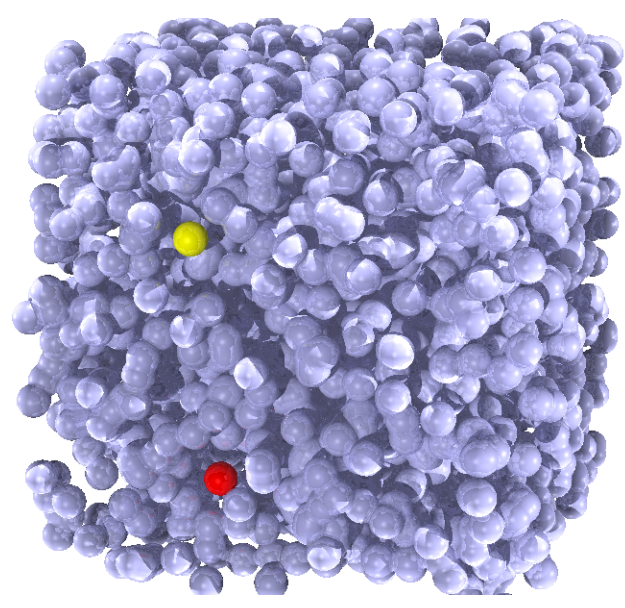

Figure 1: Typical configuration for a random array of obstacles where the void space for a tracer is close to the percolation threshold. The tracer particles (highlighted) displayed have equal radius as the obstacles and are confined to the void space.

square displacement becomes anomalous with an exponent differing from a simple fraction. The vanishing of the diffusion coefficient upon approaching the localization transition is connected to the subdiffusive motion and the divergence of the correlation length or mean cluster size. We discuss, in particular the motion of tracers that are confined to the percolating cluster in terms of the mean-square displacement, as well as the time-dependent diffusion coefficient averaged over all initial conditions of the tracers. We then elucidate the transport as response to an alternating external field in terms of the frequency-dependent conductivity and susceptibility. Furthermore, we characterize the spaceresolved dynamics in terms of the intermediate scattering function, which is accessible to scattering experiments in principle. Its long-time limit, known as the Lamb-Mößbauer factor or nonergodicity factor, reveals the trapping of particles inside the finite clusters.

\section{The Lorentz model}

A simple model for transport in disordered solids capturing all the relevant ingredients for complex transport was introduced by Lorentz already in 1905 [39]. There a single tracer or equivalently a system of particles which do not interact among themselves traverses a course of immobilized obstacles. The position of the tracer is excluded from the space occupied by the obstacles thus confined to the void space. In the simplest case hard spherical obstacles are distributed independently and randomly in space and consequently the number of obstacles $N$ per volume $V$ characterizes the emerging 
structure completely. Then a dimensionless control parameter $n^{*}=N \sigma^{d} / V$ can be introduced, where $\sigma$ is the distance of the hard-core exclusion between the tracer and a single obstacle. Note that for independent obstacles, only $\sigma$ determines the void space allowing to consider the tracer as pointlike and the obstacles as having a radius $\sigma$. Then the obstacles naturally overlap resulting in clusters of excluded space. Alternatively, one may view the obstacles as pointlike and the tracer of radius $\sigma$, reminiscent of a random Galton board. Figure 1 displays the case where the obstacles and the tracer are of the same size, with the highlighted particles moving through the frozen maze.

A self-consistent mode-coupling kinetic theory has been introduced by Götze, Leutheusser, and Yip [40, 41, 42] which provides a theory for various aspects of the dynamics of the Lorentz gas. In particular, their approximation gives an extremely accurate value for the first order density correction [43] to the Lorentz-Boltzmann value for the diffusion coefficient and correctly reflects the long-time tails in the velocity autocorrelation function (VACF) [44]. Furthermore, they correctly predict a localization transition at a critical obstacle density $n_{c}^{*}$ at which anomalous transport occurs. The competition between the critical law of the localization and the longtime tail then leads to density-dependent apparent exponents, a prediction that has been verified only recently by computer simulations [45]. Although the kinetic theory of the Lorentz model gives a remarkable description for low and moderate densities, the regime very close to the localization is more involved.

The immediate vicinity of the arrest of transport is characterized by a diverging length scale describing the distribution of clusters of the void space. Already at moderate densities the void space falls apart into disconnected finite components, hence confining the motion of the tracer particles. Thus even below the critical density, the system becomes non-ergodic in the sense that tracers initially placed in such a pocket remain there forever. Long-range transport occurs only on the infinite void space cluster percolating through the array of obstacles. At the transition point this infinite cluster ceases to exist and all tracer particles are trapped. Thus the origin of the localization is the underlying geometric percolation transition of the void space. Percolation itself has been the investigated for many years and a coherent picture has been established [32], summarized below.

Similar to a continuous phase transition, percolation exhibits a diverging length scale $\xi \sim|\epsilon|^{-\nu}$ which corresponds here to the linear dimension of the largest finite pocket. Here the reduced obstacle density $\epsilon=$ $\left(n^{*}-n_{c}^{*}\right) / n_{c}^{*}$ quantifies the distance to the critical den- sity and plays the role of a separation parameter. The weight of the infinite void space cluster also vanishes as a power law, $P_{\infty} \sim(-\epsilon)^{\beta}$ for $\epsilon<0$, as the obstacle density is increased. Directly at $n_{c}^{*}$, the infinite void space becomes fractal with a fractal dimension $d_{\mathrm{f}}=d-\beta / v$, and in coexistence with the infinite cluster, there is a self-similar hierarchy of finite clusters. In contrast to thermal phase transitions a second length scale, viz. the mean-square cluster size $\ell$, plays an important role for the percolation transition. Using scaling arguments, one can show that its divergent behavior is determined by the same two exponents as $\ell \sim|\epsilon|^{-v+\beta / 2}$. The critical exponents for percolation are known from computer simulations [32, 38] and attain the approximate values $v \approx 0.88$ and $\beta \approx 0.41$ in three-dimensional space, $d=3$.

\section{Motion on the infinite cluster}

Let us discuss first the dynamics of tracer particles that move on the percolating cluster only. Transport on the percolating cluster is expected to become fractal precisely at the critical obstacle density. After an elapsed time $t$ a particle has moved typically a distance $t^{1 / d_{\mathrm{w}}}$, where $d_{\mathrm{w}}$ is referred to as the walk dimension and attains the value of $d_{\mathrm{w}} \approx 4.81$ [38] in the three-dimensional Lorentz model. For the mean-square displacement $\delta r_{\infty}^{2}(t):=\left\langle[\mathbf{R}(t)-\mathbf{R}(0)]^{2}\right\rangle_{\infty}$ this implies $\delta r_{\infty}^{2}(t) \sim t^{2 / d_{\mathrm{w}}}$, where the index $\infty$ indicates that averaging is only for particles initially located on the infinite cluster. Below the critical obstacle density, transport should follow this subdiffusive behavior for intermediate times and then cross over to a diffusive motion $\delta r_{\infty}^{2}=6 D_{\infty} t$ for length scales larger than $\xi \sim(-\epsilon)^{-v}$, where the cluster appears homogeneous. Both aspects can be combined in the scaling law

$$
\delta r_{\infty}^{2}(t ; \epsilon)=t^{2 / d_{\mathrm{w}}} \delta \tilde{r}_{\infty}^{2}(\tilde{t}), \quad \tilde{t}=t / t_{x}
$$

i.e., all mean-square displacements have the same shape in a double-logarithmic representation. The dependence on the reduced obstacle density $\epsilon$ is solely by a change of scale $t_{x}=t_{x}(\epsilon)$. The scaling function $\delta \tilde{r}_{\infty}^{2}(\cdot)$ attains a constant for short rescaled times $\tilde{t} \ll 1$, reflecting the anomalous transport. The length scale where the crossover to diffusion occurs is given by the correlation length, which then in turn determines the crossover time to $t_{\infty} \sim \xi^{d_{\mathrm{w}}} \sim|\epsilon|^{-v d_{\mathrm{w}}}$. The crossover to diffusion is recovered by imposing $\delta \tilde{r}_{\infty}^{2}(\tilde{t}) \sim \tilde{t}^{1-2 / d_{\mathrm{w}}}$ at long rescaled times $\tilde{t} \gg 1$. As a consequence the corresponding diffusion coefficient vanishes as a power law, $D_{\infty} \sim t_{x}^{2 / d_{\mathrm{w}}-1} \sim(-\epsilon)^{v\left(d_{\mathrm{w}}-2\right)}$. Since the particles on 


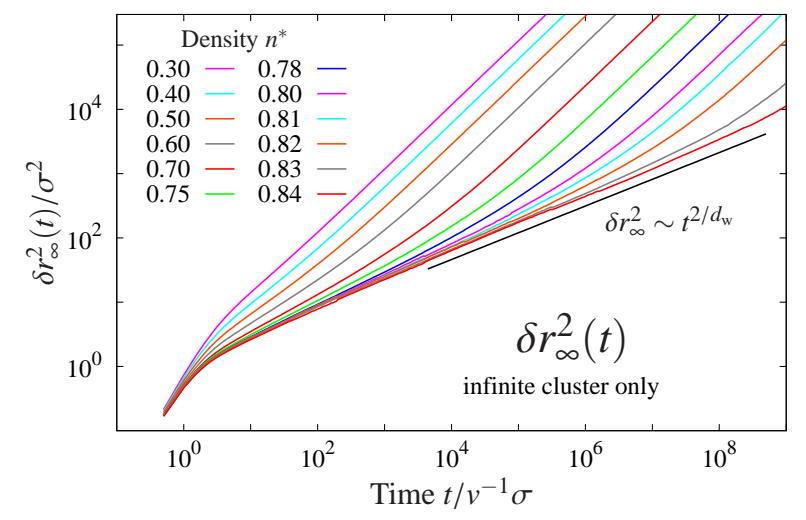

Figure 2: Double-logarithmic representation of the mean-square displacements $\delta r_{\infty}^{2}(t)$ for particles on the infinite cluster. Obstacle density increases from top to bottom. The straight line indicates anomalous transport with a walk dimension of $d_{\mathrm{w}}=4.81$.

the infinite cluster are the only ones that contribute to long-range motion, one derives the scaling behavior of the diffusion coefficient for an all-cluster-average by $D(\epsilon)=P_{\infty}(\epsilon) D_{\infty}(\epsilon) \sim(-\epsilon)^{\mu}$ with the conductivity exponent $\mu=\beta+v\left(d_{\mathrm{w}}-2\right)$.

We have performed extensive computer simulations for the Lorentz gas with ballistic particles as tracers. In this case the trajectory in configuration space consists of a series of straight lines connecting the scattering events with the obstacles. We have employed deterministic specular scattering which in particular conserves the magnitude of the velocity $v=|\mathbf{v}(t)|$. The trajectories are generated using the standard event-driven algorithm already used by Bruin [46] combined with the usual method [47, 48] for calculating correlation functions online, optimized for exponentially large time scales. The infinite cluster has been identified by a standard Voronoi tessellation to generate trajectories for the infinite-cluster-only averages.

The simulation results for the mean-square displacement $\delta r_{\infty}^{2}(t)$ are displayed in Fig. 2 for various obstacle density below the localization transition. All curves show ballistic motion $\delta r_{\infty}^{2}(t)=v^{2} t^{2}$ for times smaller than the inverse collision rate or mean collision time $\tau_{c}$. In the low-density regime, $n \rightarrow 0$, the collision time grows, $\tau_{c} \sim 1 / n \sigma^{d-1} v$, yet close to the percolation transition, the density dependence can be ignored. For low-obstacle densities the motion crosses over directly to a linear increase of $\delta r_{\infty}^{2}(t)$ in time, as is expected for normal transport. As the critical density $n_{c}^{*}$ is approached a window of subdiffusive transport emerges and the mean-square displacements increase as a power law in accordance with theoretical prediction. Directly at the critical point $n_{c}^{*} \approx 0.839$ the power law $t^{2 / d_{\mathrm{w}}}$ is observed over more than 6 six decades in time. The long-time behavior for all densities below $n_{c}^{*}$ is again diffusive but sets in at later and later times as $\epsilon \uparrow 0$. A short inspection of the curves reveals that the shapes of the crossover functions are similar and scaling behavior is anticipated to hold on a semi-quantitative level. Yet including the corrections to scaling [38] should corroborate the validity of the scaling hypothesis more generally. A more detailed analysis of the scaling behavior of $\delta r_{\infty}^{2}(t)$ can be found in [49].

\section{All-cluster-averaged motion}

Conventionally the Lorentz model considers averages over all allowed starting positions of the tracer, i.e., both on any of the finite clusters as well as on the infinite cluster. Long-range transport occurs only on the percolating cluster, but close to the threshold the finite clusters become arbitrarily large and contribute to transport at all scales. The motion of an ensemble of tracers in such a fractal landscape is also self-similar in the time-domain introducing a new exponent $z$ characteristic of the subdiffusive motion at the critical point. The unconstrained mean-square displacement is defined as $\delta r^{2}(t):=\left\langle[\mathbf{R}(t)-\mathbf{R}(0)]^{2}\right\rangle$, where the average includes initial positions in the infinite and in finite clusters according to their weight as well as over the disorder. It increases again as a power law, $\delta r^{2}(t) \sim t^{2 / z}$, where the dynamic exponent $z$ is larger than the walk dimension $d_{\mathrm{w}}$. Since the all-cluster averaged mean-square displacement has already been discussed in Refs. 37 and 50 , here we focus on the time-dependent diffusion coefficient

$$
D(t):=\frac{1}{d}\langle[\mathbf{R}(t)-\mathbf{R}(0)] \cdot \mathbf{v}(0)\rangle,
$$

i.e., the correlation of the time-dependent displacement $\mathbf{R}(t)-\mathbf{R}(0)$ with the initial velocity $\mathbf{v}(0)=\dot{\mathbf{R}}(0)$. The definition of $D(t)$ holds in any dimension $d$, in particular for $d=3$. Because of time-translational invariance the time-dependent diffusion coefficient is related to the mean-square displacement via

$$
D(t)=\frac{1}{2 d} \frac{\mathrm{d}}{\mathrm{d} t} \delta r^{2}(t) .
$$

Similarly, the velocity autocorrelation function $Z(t):=$ $(1 / d)\langle\mathbf{v}(t) \cdot \mathbf{v}(0)\rangle$ encodes the time-dependent motion via $D(t)=\int_{0}^{t} Z\left(t^{\prime}\right) \mathrm{d} t^{\prime}$ consistent with the Green-Kubo relation for the diffusion constant $D:=D(t \rightarrow \infty)$ as the long-time limit of the time-dependent diffusion coefficient. 


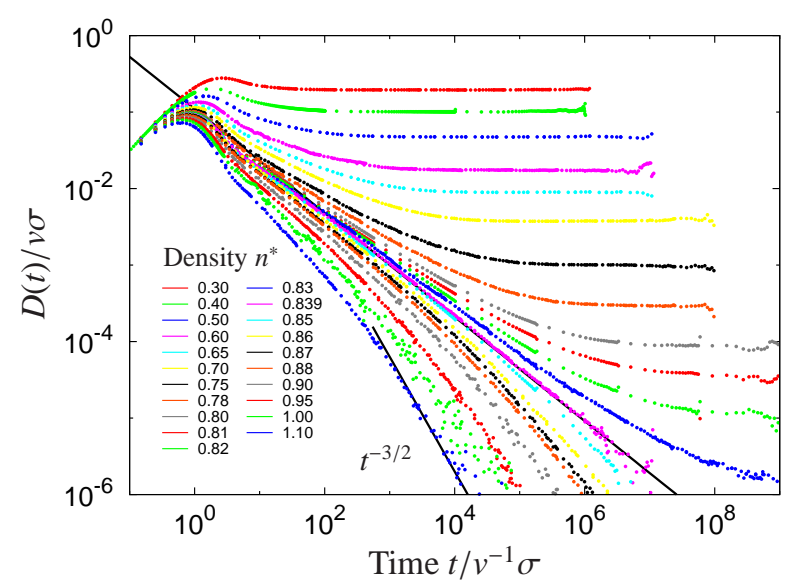

Figure 3: Double-logarithmic representation of the time-dependent diffusion coefficient $D(t)=(1 / 6) \mathrm{d} \delta r^{2}(t) / \mathrm{d} t$. Density increases from top to bottom. The full line indicates subdiffusive behavior $D(t) \sim$ $t^{2 / z-1}$ at the critical obstacle density $n_{c} \approx 0.839$. The thick line indicates the power law $t^{-3 / 2}$ characterizing the long-time decay in the localized phase.

The simulation data for the time-dependent diffusion coefficient $D(t)$ are displayed in Fig. 3. At short times, $D(t)=v^{2} t / d$ increases linearly and the curves for all scatterer densities superimpose. This reflects the free flight of the tracer until the first scattering event occurs at the collision time $\tau_{c}$. At time scales of the order of the collision time, the time-dependent diffusion coefficient displays a maximum due to the cage effect familiar from the zero crossing in the velocity autocorrelation function. For longer times, $D(t)$ decreases monotonically, effects of inertia become less and less relevant, and the structural relaxation dominates the transport.

For low and moderate obstacle densities, $D(t)$ rapidly saturates at its long-time limit $D(t \rightarrow \infty)$, which corresponds to the diffusion constant $D$ of the tracer. Upon increasing the number of scatterers towards the percolation threshold, diffusion is rapidly suppressed. In this regime $D(t)$ displays a crossover from power-law decrease to saturation and one anticipates that scaling holds. A detailed analysis of the scaling properties of the three-dimensional Lorentz model in terms of the mean-square displacement can be found in Ref. 37; the scaling of the time-dependent diffusion coefficient for a two-dimensional Lorentz model is discussed in Ref. 51

Precisely at the percolation threshold, $D(t) \sim t^{2 / z-1}$ is expected to hold for $t \rightarrow \infty$ as is inferred from the corresponding subdiffusive behavior of the mean-square displacement. Our simulations display such anomalous transport over more than five decades in time allowing the determination of the exponent $z=6.25$, con- sistent with the prediction that the universality class of the three-dimensional Lorentz model coincides with the one of a random resistor network with power-law distributed weak conductances [37, 50].

In the high-density regime, $n^{*}>n_{c}^{*}$, the timedependent diffusion coefficient vanishes in the longtime limit reflecting the fact that all tracers are trapped in finite pockets of the void space. Again, close to criticality the data follow the anomalous behavior until a crossover time and then decreases more rapidly. Yet the long-time behavior is not characterized by an exponential decay since power law distributed exit rates from cul-de-sacs lead to long-time tails in the localized phase [34]. For the three-dimensional case, the velocity autocorrelation function (VACF) is predicted to decay as $t^{-5 / 2}$ which corresponds to $t^{-3 / 2}$ for the timedependent diffusion coefficient as indicated in Fig. 3 To the best of our knowledge, this algebraic decay has not been observed before.

\section{Frequency-dependent conductivity}

In the context of ion conductors one is interested in the frequency-dependent complex conductivity $\sigma(\omega)$ which quantifies the alternating electric current density $\mathbf{j}(\omega)$ as response to an frequency-dependent homogeneous electric field $\mathbf{E}(\omega)$ in the linear regime as $\mathbf{j}(\omega)=\sigma(\omega) \mathbf{E}(\omega)$. For disordered materials that are statistically isotropic the current is parallel to the electric field and the conductivity $\sigma(\omega)$ transforms as a scalar. A decomposition into real and imaginary parts, $\sigma(\omega)=\operatorname{Re}[\sigma(\omega)]+1 \operatorname{Im}[\sigma(\omega)]$, reveals that the in-phase component $\operatorname{Re}[\sigma(\omega)] \geq 0$ describes the loss due to friction, whereas $\operatorname{Im}[\sigma(\omega)]$ encodes the storage of energy in the system. For the important case that the conducting ions can be viewed as independent, the conductivity is obtained by $\sigma(\omega)=q^{*} n_{\text {ion }} \mu(\omega)$, where $q^{*}$ is the effective charge of the ions, $n_{\text {ion }}$ their number density, and $\mu(\omega)$ the frequency-dependent mobility.

The linear response theorem extends the well-known Einstein relation $D=\mu k_{B} T$ to finite frequencies $Z(\omega)=$ $k_{B} T \mu(\omega)$, where the frequency-dependent generalization $Z(\omega)$ of the diffusion coefficient $D$ is given by

$$
Z(\omega)=\frac{1}{d} \int_{0}^{\infty}\langle\mathbf{v}(t) \cdot \mathbf{v}(0)\rangle \exp (1 \omega t) \mathrm{d} t
$$

i.e., as the one-sided Fourier transform of the velocity autocorrelation function. In particular, the GreenKubo relation for the diffusion coefficient is recovered for the stationary case, $\omega=0$. By partial integration one 


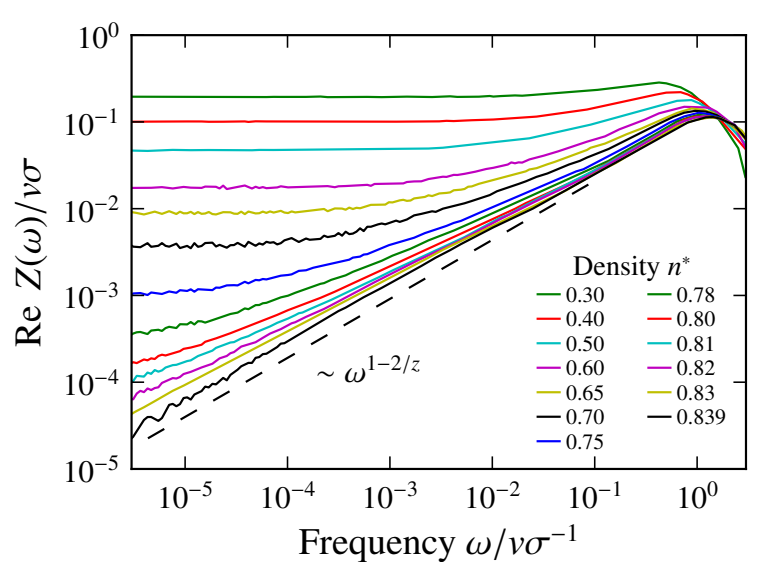

Figure 4: Frequency-dependent diffusion coefficient $\operatorname{Re}[Z(\omega)]$ on double-logarithmic scales for various obstacle densities below the localization threshold. Densities increase from top to bottom. The broken line indicates the power law $\omega^{1-2 / z}$ expected for anomalous transport at the critical density.

obtains a representation of $Z(\omega)$ in terms of the timedependent diffusion coefficient

$$
Z(\omega)=D-\mathrm{i} \omega \int_{0}^{\infty}[D(t)-D] \mathrm{e}^{\mathrm{i} \omega t} \mathrm{~d} t,
$$

with the diffusion coefficient $D=D(t \rightarrow \infty)$.

Figure 4 displays the real part of the frequencydependent diffusion coefficient, $\operatorname{Re}[Z(\omega)]$, obtained via a numerical one-side Fourier transform from our simulation data according to Eq. 5. At the critical density $n_{c}^{*}$, the power-law dependence $\operatorname{Re}[Z(\omega)] \sim \omega^{1-2 / z}$ holds over almost five decades in frequency. The fractal behavior of $Z(\omega)$ is inherited from an algebraic long-time decay of of the VACF, $Z(t) \sim t^{2 / z-2}$, or equivalently from the subdiffusive increase of the mean-square displacement, $\delta r^{2}(t) \sim t^{2 / z}$.

For obstacle densities below $n_{c}^{*}$, the curves attain a finite value at low-frequencies which is identified with the diffusion constant $D=Z(\omega=0)$. At high frequencies, the frequency-dependent diffusion coefficient approaches the critical law. The two regimes of anomalous transport and diffusion merge at a characteristic crossover frequency $\omega_{x}$ which is shifted to lower values as the critical density is approached.

These observations suggest the scaling behavior

$$
Z(\omega ; \epsilon)=(-1 \omega)^{1-2 / z} \mathcal{Z}_{ \pm}(\hat{\omega}), \quad \hat{\omega}=\omega / \omega_{x},
$$

in the close vicinity of the localization transition. Here the scaling functions $\mathcal{Z}_{+}(\cdot)$ and $\mathcal{Z}_{-}(\cdot)$ refer to densities above (+) and below (-) the critical density, respectively. For large rescaled frequencies $\hat{\omega} \gg 1$, the tracers

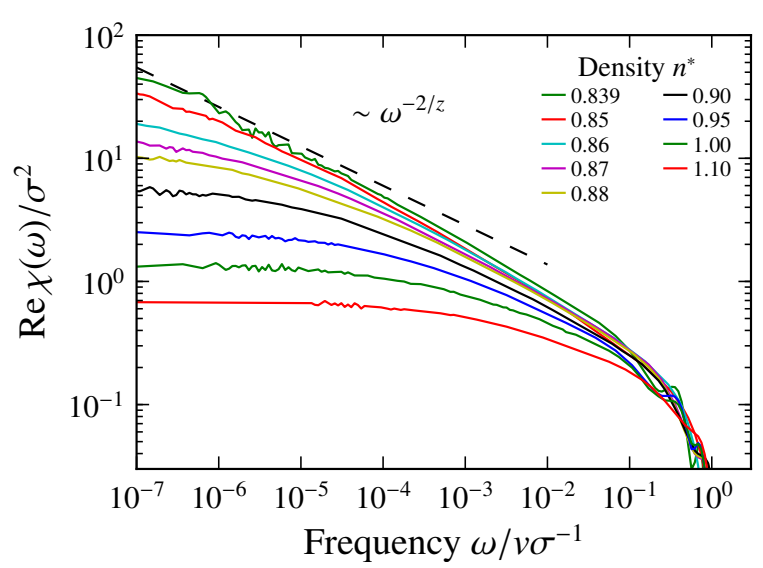

Figure 5: Frequency-dependent susceptibility $\operatorname{Re}[\tilde{\chi}(\omega)]$ on doublelogarithmic scales for various obstacle densities above the localization threshold. Densities increase from top to bottom. The broken line indicates the power-law divergence $\omega^{-2 / z}$ expected for anomalous transport at the critical density.

cannot explore the entire landscape and transport is fractal, $\mathcal{Z}_{ \pm}(\hat{\omega} \rightarrow \infty) \rightarrow$ const, i.e., indistinguishable from the critical point. In particular, the constant is the same on both sides of the transition.

On the conducting side, $\mathcal{Z}_{-}(\hat{\omega}) \sim(-1 \hat{\omega})^{2 / z-1}$ for $\hat{\omega} \rightarrow$ 0 is enforced by the requirement of a finite conductivity, which in turn implies the scaling law $D(\epsilon) \sim \omega_{x}^{1-2 / z}$. Defining the conductivity exponent $\mu$ via $D(\epsilon) \sim(-\epsilon)^{\mu}$ yields $\omega_{x} \sim(-\epsilon)^{\mu z /(z-2)}$.

On the insulating side, it is advantageous to discuss the complex polarizability $\chi(\omega)=\sigma(\omega) /(-1 \omega)$ rather than the conductivity itself. Let us define the susceptibility $\tilde{\chi}(\omega)=Z(\omega) /(-1 \omega)$ by dividing out factors that are irrelevant for the present discussion, $\chi(\omega)=$ $\left(q^{*} n_{\text {ion }} / k_{B} T\right) \tilde{\chi}(\omega)$. Then $\tilde{\chi}(\omega)$ has the dimension of the square of a length. A finite polarizability is obtained by imposing $\mathcal{Z}_{+}(\hat{\omega}) \sim(-1 \hat{\omega})^{2 / z}$ for low frequencies $\hat{\omega} \ll 1$. The static susceptibility diverges upon approaching the critical obstacle density $\tilde{\chi}(\omega=0, \epsilon) \sim \omega_{x}^{-2 / z}$. Because of geometrical reasons this susceptibility should diverge in the same way as the mean-square cluster size $\tilde{\chi}(\omega=0, \epsilon) \sim \ell^{2} \sim \epsilon^{-(2 v-\beta)}$. Combining the preceding arguments reveals the scaling relation between the conductivity exponent and dynamic exponent $z$ to $z=(2 v-\beta+\mu) /(v-\beta / 2)$. Then one also observes that the crossover frequency scales as $\omega_{x} \sim t_{x}^{-1}$, i.e the inverse of the crossover time $t_{x}$ of the mean-square displacement for the infinite cluster only.

The simulation results for the susceptibility on the insulating side are displayed in Fig. 5. As discussed above, the real part becomes finite in the static case reflecting the finite polarizability of the system. Ap- 


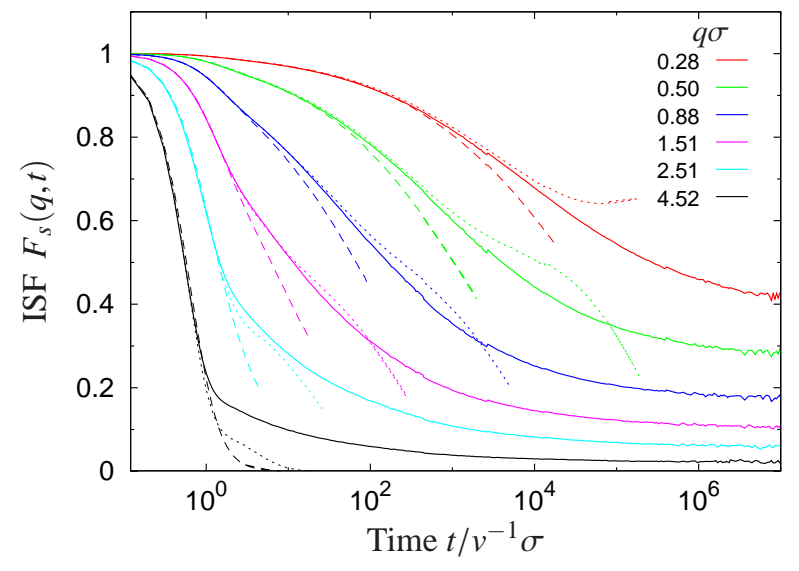

Figure 6: Self-intermediate scattering function $F_{s}(q, t)$ on a logarithmic time scale at the critical density $n_{c}^{*}=0.839$. Wavenumbers increase from top to bottom. The full lines correspond to the simulation data, the broken ones to the Gaussian approximation $F_{s}^{\text {Gauss }}(q, t)$, and the dotted ones include the first non-Gaussian correction $F_{s}^{\mathrm{NGP}}(q, t)$.

proaching the delocalization transition by diluting the obstacles gives rise to a strong increase of the static polarizability. At the critical density, the susceptibility displays power-law behavior in the frequency with finite response only for alternating external electric fields.

\section{Self-intermediate scattering function}

Spatio-temporal information on the dynamics of the tracer can be extracted by considering the selfintermediate scattering function (ISF) defined by

$$
F_{s}(q, t):=\langle\exp (\mathbf{1} \mathbf{q} \cdot \Delta \mathbf{R}(t)\rangle .
$$

Thus $F_{s}(q, t)$ is the characteristic function of the displacements $\Delta \mathbf{R}(t):=[\mathbf{R}(t)-\mathbf{R}(0)]$ considered as the random variable. We have anticipated already that due to statistical isotropy $F_{s}(q, t)$ does not depend on the direction of the wavevector $\mathbf{q}$, but only on its magnitude $q=|\mathbf{q}|$. The intermediate scattering function encodes all moments of the displacement via derivatives, for instance the mean-square displacement can be obtained as $\delta r^{2}(t)=-d \lim _{q \rightarrow 0} \partial^{2} F_{s}(q, t) / \partial q^{2}$. Scattering techniques such as neutron-spin echo or photon correlation spectroscopy have direct access to the ISF and there $\hbar \mathbf{q}$ plays the role of the momentum transfer from the tracer to the neutrons or photons. The simulation results for the ISF for small to moderate wavenumbers $q$ for obstacle density directly at $n_{c}^{*}$ are displayed in Fig. 6 As expected transport becomes slower on larger length scales. In contrast to glassy dynamics characterized by a two-step relaxation process, here no plateau develops at intermediate times. Rather all intermediate scattering function appear to saturate at some finite level. Since the infinite cluster has non-extensive weight at the critical point [32] its dynamics does not contribute to the ISF shown. Yet the infinite hierarchy of finite clusters coexisting with the percolation cluster leads to non-ergodic behavior as manifested in the ISF, see below. Since the finite clusters are present even below the critical density, one should in fact expect non-ergodic behavior due to the falling apart of the configuration space at all finite densities.

The spatio-temporal information encoded in the ISF is revealed by gradually changing the wavenumber $q$. To highlight the non-trivial behavior we have included the Gaussian approximation $(d=3)$

$$
F_{s}^{\text {Gauss }}(q, t)=\exp \left(-q^{2} \delta r^{2}(t) / 6\right),
$$

in Fig. 6 This approach ignores all cumulants except for the second, which reduces to the mean-square displacement $\delta r^{2}(t)$. The Gaussian model accounts approximately for the increase of time scale upon changing the wavenumber and also provides a quantitative description of the initial decay of the ISF. Interestingly the Gaussian model becomes worse on increasing the length scale, demonstrating the fact that transport at the critical density never becomes Gaussian even at the largest scales. A similar observation on the planar Lorentz model has been made recently in the context of fluorescence correlation spectroscopy [52]. Including also the first non-Gaussian parameter (NGP),

$$
\alpha_{2}(t):=\frac{3}{5}\left\langle\left[\Delta \mathbf{R}(t)^{2}\right]^{2}\right\rangle\left\langle\left\langle\Delta \mathbf{R}(t)^{2}\right\rangle^{2}-1,\right.
$$

in the intermediate scattering function,

$$
F_{s}^{\mathrm{NGP}}(q, t)=\mathrm{e}^{-q^{2} \delta r^{2}(t) / 6}\left\{1+\frac{1}{2} \alpha_{2}(t)\left[\frac{q^{2} \delta r^{2}(t)}{6}\right]^{2}\right\},
$$

describes the simulation data even to times larger by a factor of 10. Nevertheless, this approximation too fails on a qualitative level for long times since it predicts a decay of all ISFs to zero due to the unbounded increase of the mean-square displacement.

\section{Lamb-Mössbauer factor}

The long-time limit of the intermediate scattering function

$$
f_{s}(q):=\lim _{t \rightarrow \infty} F_{s}(q, t)
$$


is referred to as Lamb-Mössbauer factor or nonergodicity parameter, also known as the EdwardsAnderson parameter in the spin glass community [1]. A non-vanishing $f_{s}(q)$ indicates that dynamic correlations are persistent forever implying that the dynamics is nonergodic. Roughly speaking $f_{s}(q)$ measures the fraction of particles that are trapped on length scale $2 \pi / q$. For the Lorentz model the presence of finite clusters at all densities suggests that the non-ergodicity transition has to be disentangled from the localization transition. A clear distinction can be made by considering the longwavelength limit $f_{s}(0)=\lim _{q \rightarrow 0} f_{s}(q)$ of the LambMössbauer factor, since $1-f_{s}(0)=P(\epsilon)$ where $P(\epsilon)$ denotes the weight of the percolating cluster. Since above the localization threshold $n^{*}>n_{c}^{*}$ the infinite cluster ceases to exist, $f_{s}(0)$ attains the value unity, whereas increasing the obstacle density from below leads to an expected critical behavior $1-f_{s}(0) \sim(-\epsilon)^{\beta}$.

For finite wavenumbers this sharp transition is rounded off and crossover scaling should hold [36]. A convenient form is obtained by postulating $1-f_{s}(q ; \epsilon)=$ $|\epsilon|^{\beta} \hat{f}_{ \pm}(q \xi)$, where the scaling functions $\hat{f}_{ \pm}$refer to densities above the threshold $(+)$ and the below the localization transition (-). The critical behavior of the long-wavelength limit is recovered by requiring $\hat{f}_{-}(x \rightarrow$ $0)=$ const. , and $\hat{f}_{+}(x \rightarrow 0)=0$, respectively. For large wavenumbers and densities close to the transition the dynamics cannot resolve the finiteness of the correlation length and should become independent of the separation parameter, which is achieved by imposing $\hat{f}_{ \pm}(x) \sim x^{\beta / n u}$. This in turn implies a singular dependence of the Lamb-Mössbauer factor on the wavenumber $1-f_{s}(q ; \epsilon=0) \sim q^{\beta / v}$ directly at the threshold.

The Lamb-Mössbauer factors obtained from our simulation results for the ISF as extrapolation to infinite times are displayed in Fig. 7. Upon increasing the density $f_{s}(q)$ increases monotonically and the shape develops into a rapid crossover for small wavenumbers. Yet for finite wavenumber nothing peculiar happens at the percolation threshold. Only the extrapolation to the long wavelength limit indicates the localization transition in the sense that it converges to unity rapidly as $n_{c}^{*}$ is approached from below. Due to the large statistical uncertainties we have not attempted to validate the scaling law. Nevertheless the approach of the long-wavelength limit towards unity is compatible with the power law expected for the weight of the infinite cluster.

\section{Summary and Conclusion}

Transport in disordered material as studied in terms of the Lorentz model displays many facets that go be-

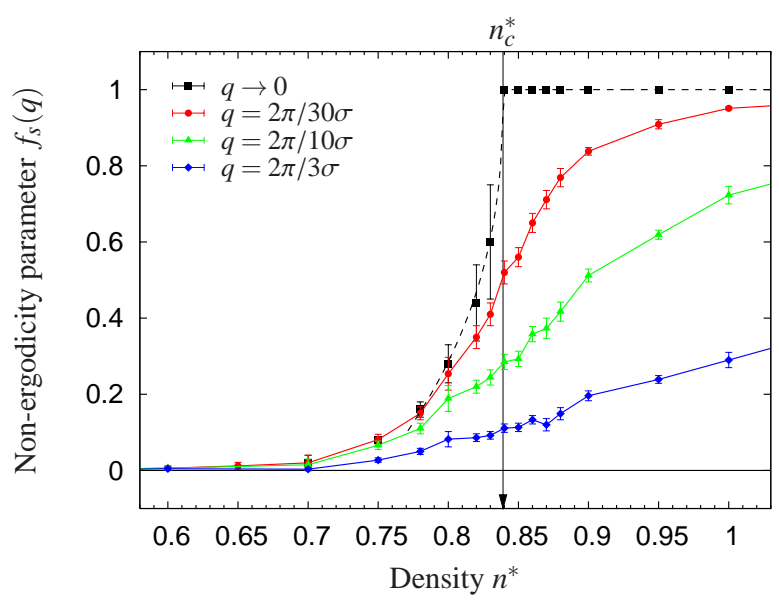

Figure 7: Lamb-Mößbauer factor $f_{s}(q)=F_{s}(q, t \rightarrow \infty)$ as a function of the obstacle density $n^{*}$. As the wavenumber decreases the curves steepen. The extrapolation to zero wavenumber exhibits a sharp transition at the critical obstacle density $n_{c}^{*}$. The broken line corresponds to a power law $(-\epsilon)^{\beta}$ and serves as a guide to the eye.

yond the simple subdiffusive increase of the meansquare displacement. We have elucidated some of these aspects as they are manifest in the mean-square displacement for tracers that are confined to the percolating cluster only, the time-dependent diffusion coefficient for an all-cluster average, the frequency-dependent conductivity and susceptibility in response to an $A C$ electric field, as well as the intermediate scattering function and its long-time limit, the Lamb-Mössbauer factor.

The percolation transition of the underlying void space induces a self-similar geometric fractal with anomalous transport at the localization threshold. The interplay of time and length scales leads to a striking behavior of the complex transport properties many aspects of which can be rationalized in terms of scaling laws. The scaling predictions require three independent exponents as input and we have demonstrated that all three values can be revealed by considering different limits of certain two-time correlation functions. In particular, the detailed study shows that simplified concepts such as fractional Brownian motion or continuous time random walks (CTRW) cannot explain the dynamics as soon as spatio-temporal information beyond the mean-square displacement at the threshold is studied.

The localization transition in the Lorentz model in its simplest variant has now been studied from various aspects and nice agreement of extensive computer simulations with theoretical notions has been achieved. An open question is how anomalous transport occurs in a class of glass-forming liquids that displays a strong separation of time scales, and a possible route to an 
answer is to include more microscopic details into the Lorentz model, thereby gradually adding more complexity. These changes could include studying the motion of a tracer in a frozen matrix of correlated obstacles generated from a snapshot of a real liquid. In particular, one has to clarify the role of the narrow gaps that emerge in the frozen labyrinth and their influence on the value of the dynamic or conductivity exponent. Furthermore, it is interesting to investigate the effect of interacting tracers inside the quasi-arrested host matrix since for long times the tracers will strongly interact in the ramified network of channels. A further interesting aspect is the interaction of a dynamic maze on the particle, i.e., the effect of vibrations of the slow component on the dynamics of the tracer.

\section{Acknowledgments}

Thomas Franosch is indebted to Wolfgang Götze for many years of discussions and his priceless insight into complex transport connected to the glass and localization transition.

Financial support from the Deutsche Forschungsgemeinschaft via contract No. FR $850 / 6-1$ is gratefully acknowledged. This project is supported by the German Excellence Initiative via the program "Nanosystems Initiative Munich (NIM)."

\section{References}

[1] W. Götze, Complex Dynamics of Glass-Forming Liquids - A Mode-Coupling Theory, Oxford, 2009.

[2] W. Götze, L. Sjögren, General properties of certain nonlinear integrodifferential equations, J. Math. Anal. Appl. 195 (1995) 230-250.

[3] T. Franosch, T. Voigtmann, Completely monotone solutions of the mode-coupling theory for mixtures, J. Stat. Phys. 109 (2002) 237.

[4] T. Franosch, W. Götze, Relaxation rate distributions for supercooled liquids, J. Phys. Chem. B 103 (20) (1999) 4011-4017.

[5] W. van Megen, S. M. Underwood, Dynamic-light-scattering study of glasses of hard colloidal spheres, Phys. Rev. E 47 (1) (1993) 248-261.

[6] W. van Megen, T. C. Mortensen, S. R. Williams, J. Müller, Measurement of the self-intermediate scattering function of suspensions of hard spherical particles near the glass transition, Phys. Rev. E 58 (5) (1998) 6073-6085.

[7] M. Sperl, Nearly logarithmic decay in the colloidal hard-sphere system, Phys. Rev. E 71 (6) (2005) 060401.

[8] K. N. Pham, A. M. Puertas, J. Bergenholtz, S. U. Egelhaaf, A. Moussaid, P. N. Pusey, A. B. Schofield, M. E. Cates, M. Fuchs, W. C. K. Poon, Multiple glassy states in a simple model system, Science 296 (5565) (2002) 104-106.

[9] K. Dawson, G. Foffi, M. Fuchs, W. Götze, F. Sciortino, M. Sperl, P. Tartaglia, T. Voigtmann, E. Zaccarelli, Higher-order glasstransition singularities in colloidal systems with attractive interactions, Phys. Rev. E 63 (1) (2000) 011401.
[10] G. Li, W. Du, X. Chen, H. Cummins, N. Tao, Testing Modecoupling Predictions for alpha-Realaxation and beta-Relaxation in Ca0.4K0.6(NO3)1.4 near the Liquid-Glass Transition by Light-Scattering, PHYSICAL REVIEW A 45 (6) (1992) 38673879.

[11] T. Franosch, W. Götze, M. R. Mayr, A. P. Singh, Evolution of structural relaxation spectra of glycerol within the gigahertz band, Phys. Rev. E 55 (3) (1997) 3183-3190.

[12] A. Singh, G. Li, W. Götze, M. Fuchs, T. Franosch, H. Cummins, Structural relaxation in orthoterphenyl: a schematic-modecoupling-theory-model analysis, J. Non-Cryst. Solids 235-237 (1998) 77.

[13] G. Foffi, W. Götze, F. Sciortino, P. Tartaglia, T. Voigtmann, Mixing effects for the structural relaxation in binary hard-sphere liquids, Phys. Rev. Lett. 91 (8) (2003) 085701.

[14] W. Götze, T. Voigtmann, Effect of composition changes on the structural relaxation of a binary mixture, Phys. Rev. E 67 (2) (2003) 021502

[15] R. Schilling, T. Scheidsteger, Mode coupling approach to the ideal glass transition of molecular liquids: Linear molecules, Phys. Rev. E 56 (3) (1997) 2932-2949.

[16] T. Franosch, M. Fuchs, W. Götze, M. Mayr, A. Singh, Theory for the reorientational dynamics in glass-forming liquids, Phys. Rev. E 56 (5) (1997) 5659-5674.

[17] R. Schilling, Reference-point-independent dynamics of molecular liquids and glasses in the tensorial formalism, Phys. Rev. E 65 (5) (2002) 051206.

[18] S.-H. Chong, W. Götze, Idealized glass transitions for a system of dumbbell molecules, Phys. Rev. E 65 (4) (2002) 041503.

[19] M. Fuchs, M. E. Cates, Theory of nonlinear rheology and yielding of dense colloidal suspensions, Phys. Rev. Lett. 89 (24) (2002) 248304.

[20] J. M. Brader, M. E. Cates, M. Fuchs, First-principles constitutive equation for suspension rheology, Physical Review Letters 101 (13) (2008) 138301.

[21] A. Meyer, J. Horbach, W. Kob, F. Kargl, H. Schober, Channel formation and intermediate range order in sodium silicate melts and glasses, Phys. Rev. Lett. 93 (2) (2004) 027801.

[22] T. Voigtmann, J. Horbach, Slow dynamics in ion-conducting sodium silicate melts: Simulation and mode-coupling theory, Europhys. Lett. 74 (3) (2006) 459-465.

[23] A. J. Moreno, J. Colmenero, Anomalous dynamic arrest in a mixture of large and small particles, Physical Review E (Statistical, Nonlinear, and Soft Matter Physics) 74 (2) (2006) 021409.

[24] N. Kikuchi, J. Horbach, Mobile particles in an immobile environment: Molecular dynamics simulation of a binary yukawa mixture, EPL 77 (2007) 2600.

[25] J. Kurzidim, D. Coslovich, G. Kahl, Single-particle and collective slow dynamics of colloids in porous confinement, Phys. Rev. Lett. 103 (13) (2009) 138303.

[26] K. Kim, K. Miyazaki, S. Saito, Slow dynamics in random media: Crossover from glass to localization transition, EPL 88 (3) (2009) 36002.

[27] V. Krakoviack, Liquid-glass transition of a fluid confined in a disordered porous matrix: A mode-coupling theory, Phys. Rev. Lett. 94 (6) (2005) 065703.

[28] V. Krakoviack, Mode-coupling theory for the slow collective dynamics of fluids adsorbed in disordered porous media, Phys. Rev. E 75 (3) (2007) 031503.

[29] T. Voigtmann, J. Horbach, Double transition scenario for anomalous diffusion in glass-forming mixtures, Phys. Rev. Lett. 103 (20) (2009) 205901.

[30] V. Krakoviack, Tagged-particle dynamics in a fluid adsorbed in a disordered porous solid: Interplay between the diffusionlocalization and liquid-glass transitions, Phys. Rev. E 79 (6) 
(2009) 061501.

[31] S. Papenkort, F. Höfling, T. Franosch, T. VoigtmannIn preparation.

[32] D. Stauffer, A. Aharony, Introduction to Percolation Theory, 2nd Edition, Taylor \& Francis, London, 1994.

[33] J. P. Straley, Non-universal threshold behaviour of random resistor networks with anomalous distributions of conductances, J. Phys. C 15 (1982) 2343-2346.

[34] J. Machta, S. M. Moore, Diffusion and long-time tails in the overlapping Lorentz gas, Phys. Rev. A 32 (1985) 3164.

[35] B. I. Halperin, S. Feng, P. N. Sen, Differences between lattice and continuum percolation transport exponents, Phys. Rev. Lett. 54 (1985) 2391-2394.

[36] J. Kertész, J. Metzger, Properties of the density relaxation function in classical diffusion models with percolation transition, J. Phys. A 16 (1983) L735-L739.

[37] F. Höfling, T. Franosch, E. Frey, Localization transition of the three-dimensional Lorentz model and continuum percolation, Phys. Rev. Lett. 96 (2006) 165901.

[38] A. Kammerer, F. Höfling, T. Franosch, Cluster-resolved dynamic scaling theory and universal corrections for transport on percolating systems, EPL 84 (2008) 66002.

[39] H. A. Lorentz, Le mouvement des electrons dans les metaux, Arch. Néerl. Sci. Exact Natur. 10 (1905) 336-370.

[40] W. Götze, E. Leutheusser, S. Yip, Dynamical theory of diffusion and localization in a random, static field, Phys. Rev. A 23 (1981) 2634.

[41] W. Götze, E. Leutheusser, S. Yip, Correlation functions of the hard-sphere Lorentz model, Phys. Rev. A 24 (1981) 1008.

[42] W. Götze, E. Leutheusser, S. Yip, Diffusion and localization in the two-dimensional Lorentz model, Phys. Rev. A 25 (1982) 533.

[43] A. Weijland, J. M. J. van Leeuwen, Non-analytic density behaviour of the diffusion coefficient of a Lorentz gas II, Physica (Amsterdam) 38 (1968) 35.

[44] M. H. Ernst, A. Weijland, Long time behaviour of the velocity auto-correlation function in a Lorentz gas, Phys. Lett. A 34 (1971) 39.

[45] F. Höfling, T. Franosch, Crossover in the slow decay of dynamic correlations in the Lorentz model, Phys. Rev. Lett. 98 (14) (2007) 140601.

[46] C. Bruin, Logarithmic terms in the diffusion coefficient for the Lorentz gas, Phys. Rev. Lett. 29 (25) (1972) 1670-1674.

[47] D. Frenkel, B. J. Smit, Understanding Molecular Simulation, 2nd Edition, Academic Press, London, 2001

[48] P. H. Colberg, F. Höfling, Accelerating glassy dynamics on graphics processing units, arXiv:0912.3824 [physics.comp-ph] (2009).

[49] M. Spanner, Transport in the correlated lorentz model, Master's thesis, Friedrich-Alexander-Universität Erlangen Nürnberg (2010).

[50] F. Höfling, T. Munk, E. Frey, T. Franosch, Critical dynamics of ballistic and Brownian particles in a heterogeneous environment, J. Chem. Phys. 128 (16) (2008) 164517.

[51] T. Bauer, F. Höfling, T. Munk, E. Frey, T. Franosch, Localization transition in the two-dimensional Lorentz model, arXiv:1003.2918 [cond-mat.soft].

[52] F. Höfling, K.-U. Bamberg, T. Franosch, Anomalous transport resolved in space and time by fluorescence correlation spectroscopy, arXiv:1003.3762 [cond-mat.soft]. 